\title{
The Evaluation Model of "Sunshine" Sports Based on Fuzzy AHP in University
}

\author{
Ma Yan ${ }^{*}$ and Ding Bin*
}

Heilongjiang Bayi Agricultural University, Heilongjiang Daqing, 163319 China

\begin{abstract}
Teaching quality evaluation system is a multi-level and multi-target complex issue, with numerous evaluation indexes, and to take all evaluation indexes as input of neural network would cause complex structure of neural network, thus affecting the performance of teaching quality evaluation system. This paper has proposed a teaching quality evaluation method based on AHP-BPNN. Firstly, the AHP is used to sort importance of evaluation index system, and then indicators that have an important impact on evaluation results are filtered out as input of BPNN, and finally, evaluation model is established using NN. Simulation experiment is made in the case of "Data Structure" in Fuyang Normal College, and the simulation results show that, AHP-BPNN not only simplifies the structure of the neural network, but also improves the evaluation accuracy and efficiency of the teaching quality, thus it is a feasible and effective method of teaching quality evaluation.
\end{abstract}

Keywords: AHP, artificial NN, evaluation model, teaching quality.

\section{INTRODUCTION}

Teaching quality evaluation is a crucial part of teaching quality management in school, and the evaluation of the teaching quality can promote the reform of teaching method and content [1], improving quality and level of teaching. However, the teaching quality evaluation is a tedious statistical process of heavy workload, thus to make scientific and accurate evaluation of the teaching quality is a difficult issue which is also worthy of study $[2,3]$.

Teaching quality evaluation has been valued by the majority of universities and educational management, and the artificial methods are used in traditional evaluation methods. With strong subjectivity, its evaluation precision is relatively low, unable to fully reflect the true level of teaching quality [4]. Subsequently, there has been the teaching quality evaluation of expert system, which has improved the accuracy of teaching quality evaluation to some extent, but the teaching quality assessment is a multi-target and multi-level evaluation, involving many indicators and contents, and choices of evaluation index are affected by personal preferences, level of education and other factors [5]. It is difficult to exclude the error caused by human factors, leading to strong subjectivity and low reliability in evaluation results. In recent years, with the rapid development of information technology, the teaching quality evaluation methods appear based on multiple linear regression, partial least squares, support vector machines, neural networks and others $[6,7]$.

Multiple linear regression and partial least square is to make evaluations based on linear relationship, unable to best approximate nonlinear problems. The relationship between evaluation indexes of teaching quality and between the evaluation results complex is complex and nonlinear, thus the linear evaluation method is limited in applications. Support vector machine is used for small sample. When the sample is large, the training time is long, and the operating efficiency is low. However, artificial neural network has merits of nonlinearity, real-time optimization, intelligent learning, etc., thus it becomes the main method of automatic evaluation of teaching quality currently.

In order to further improve the accuracy of the teaching quality, an AHP-BPNN teaching quality evaluation method is proposed [8]. Simulation results show that compared to other evaluation methods, AHP-BPNN effectively improves the evaluation accuracy of teaching quality, and the evaluation results can better reflect the authenticity of teachers' teaching level $[9,10]$.

\section{TEACHING QUALITY EVALUATION MODEL BASED ON AHP-BPNN}

\subsection{Building of Teaching Quality Evaluation System}

Teaching quality assessment is subject to a variety of factors, such as teaching method, teaching attitude, teaching content, classroom management, teaching effectiveness, thus scientific and accurate establishment of teaching quality evaluation system is the basis to obtain the evaluation results of high accuracy. Through systematic analysis and experts' commentary, with reference to the relevant literature and research, and AHP is used to establish teaching quality evaluation system shown in.

As can be seen from Fig. (1), the evaluation index domain $\mathrm{V}=$ (teaching method, teaching attitude, teaching content, teaching effect), and each evaluation index includes a plurality of sub-indexes. Teaching quality evaluation is di- 


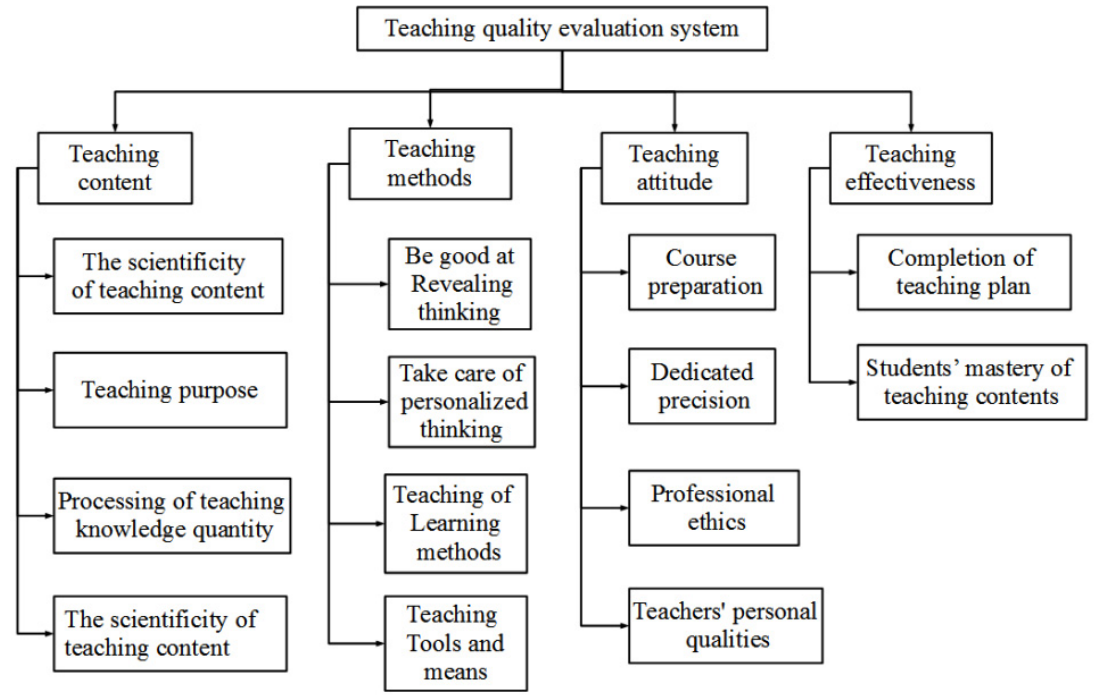

Fig. (1). Index system of teaching quality evaluation.

vided into five grades: excellent, good, middle, poor and worse.

\subsection{AHP Index Screening}

(1) Construction of comparison matrix of indicator

Construction of comparison matrix of indicator is a key step on AHP. To reduce the influence of subjective factors, comparison between the two items of teaching quality evaluation index is made, and judgment matrix A is constructed, and element values in matrix A indicate the relative importance of evaluation index on teaching quality evaluation results. In this paper, determination is made by joint rating from teaching departments and experts who are familiar classroom teaching quality, and assignment standard of elements in judgment matrix is as shown in Table $\mathbf{1}$.

Table 1. Assignment standard of elements in comparison matrix.

\begin{tabular}{|c|c|}
\hline Assignment (wi / wj) & Explanation \\
\hline \hline 1 & $\begin{array}{c}\text { Indicates that two indicators have the same } \\
\text { importance }\end{array}$ \\
\hline 3 & $\begin{array}{c}\text { Indicate that index } \mathrm{Vi} \text { is slightly more impor- } \\
\text { tant than } \mathrm{Vj}\end{array}$ \\
\hline 5 & $\begin{array}{c}\text { Indicate that index Vis is clearly more im- } \\
\text { portant than } \mathrm{Vj}\end{array}$ \\
\hline 7 & $\begin{array}{c}\text { Indicate that index } \mathrm{Vi} \text { is strongly more im- } \\
\text { portant than } \mathrm{Vj}\end{array}$ \\
\hline 9 & $\begin{array}{c}\text { Indicate that index } \mathrm{Vi} \text { is extremely more } \\
\text { important than } \mathrm{Vj}\end{array}$ \\
\hline
\end{tabular}

(2) Weight computing of evaluation index and consistency check

Based on the evaluation-factors index matrix, first of all, we can obtain $\mathrm{W}$ through $A W=\lambda_{\max } W$, then performing normalization processing, and the relative importance weights of corresponding index to the previous level are ob- tained, and finally, we conduct consistency check of comparison matrix.

The relative importance of the same level to the overall evaluation results of teaching quality is calculated to figure out comprehensive weight, and then consistency check is performed in comparison matrix from higher to lower layers.

Finally, evaluation index is sorted according to the weight of teaching quality indicator [11].

\section{(3) The sieving of important index}

According to the order of affecting weight of teachingquality final evaluation results for each index, unimportant index is excluded, and the relatively important index is screened as input of BPNN to reduce the input dimension of neural network, simplifying network results, so as to speed up the learning speed of neural networks, thus improving accuracy and efficiency of teaching quality evaluation.

\subsection{BPNN Model}

BPNN is an error back-propagation neural network, constituted of input layer, hidden layer and output layer, becoming the most widely used artificial neural network.

Output of BPNN input layer is [12]:

$O_{j}^{(1)}=x(j)$

Input and output of NN hidden layer is:

$$
\left\{\begin{array}{l}
n e t_{i}^{(2)}(k)=\sum_{j=0}^{M} w_{i j}^{(2)} O_{j}^{(1)} \\
O_{i}^{(2)}(k)=f\left(n e t_{i}^{(2)}(k)\right)
\end{array}\right.
$$

Activation function of neuron in hidden layer approached by symmetric positive and negative Sigmoid function is:

$$
f(x)=\tanh (x)=\frac{e^{x}-e^{-x}}{e^{x}+e^{-x}}
$$




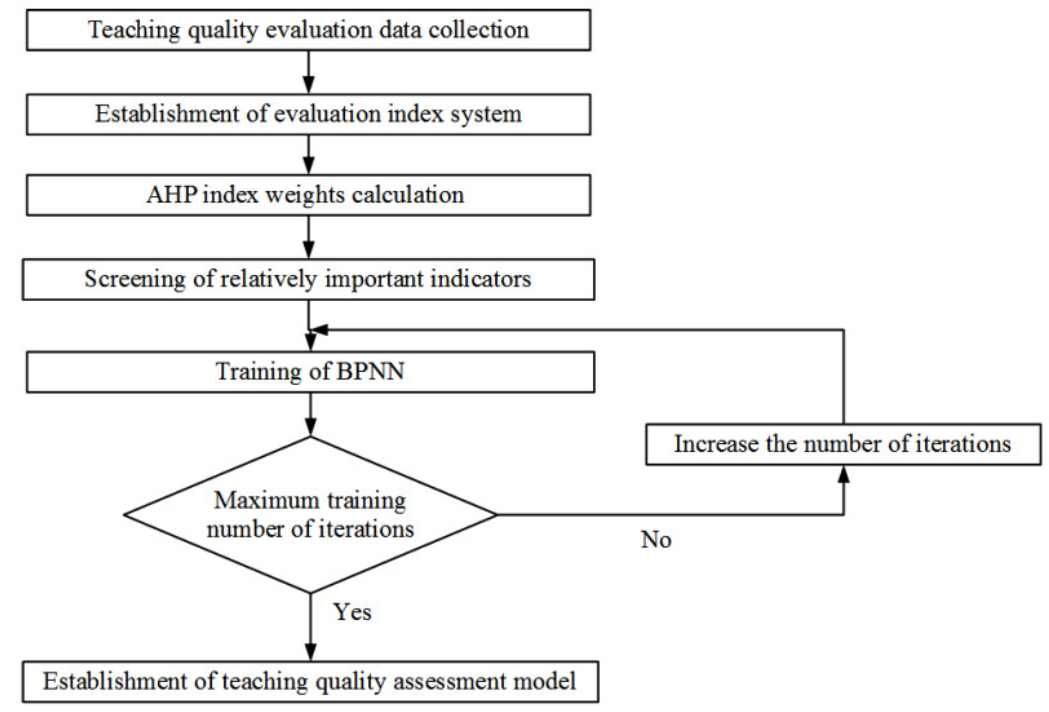

Fig. (2). AHP-BPNN teaching quality evaluation processes.

Input and output of network output layer:

$$
\left\{\begin{array}{l}
n e t_{l}^{(3)}(k)=\sum_{i=0}^{Q} w_{l i}^{(3)} O_{i}^{(2)}(k) \\
O_{l}^{(3)}(k)=g\left(n e t_{l}^{(3)}(k)\right)
\end{array}\right.
$$

Activation function of neuron in output layer approached by non-negative Sigmoid function is:

$g(x)=\frac{e^{x}}{e^{x}+e^{-x}}$

Gradient descent algorithm is adopted to determine the threshold and weight of BPNN, so that the mean square error indicators of teaching quality evaluation can reach the minimum.

\subsection{AHP-BPNN Teaching Quality Evaluation Process}

(1) According to the actual requirements of the expert system, the front line teachers and teaching quality evaluation, AHP is employed to establish the stratified index system architecture of teaching quality evaluation.

(2) AHP is used to calculate comprehensive weight of teaching evaluation index, and sorting is conducted according to the importance of the index weights.

(3) Based on the evaluation index weight, and using the screening method, evaluation index that has a major impact on the results of teaching quality evaluation is chosen.

(4) According to the evaluation index screened by AHP, the number of neurons in BPNN output layer is determined, and the gradation of teaching quality evaluation is taken as the output of model. The number of neurons in the hidden layer can be determined by the method of gradual growth, thus determining topology of BPNN model.

(5) Normalization processing of index is performed to eliminate the adverse effects dimensional differences of index.
(6) BPNN parameters are initialized, and adequate sample of teaching quality evaluation is selected, and then training and learning is conducted using BPNN, in order to establish the teaching quality evaluation model.

(7) The established evaluation model is employed to evaluate teachers' teaching quality to be evaluated, with evaluation results output, and its performances are analyzed. AHP-BPNN teaching quality evaluation process is shown in Fig. (2).

\section{THE APPLICATION OF AHP-BPNN IN TEACHING QUALITY EVALUATION}

\subsection{Data Sources}

To test the performance of teaching quality assessment based on AHP-BPNN algorithm, simulation experiment is made in the teaching quality evaluation data of "Data Structure" in Heilongjiang Bayi Agricultural University. With a total of 5000 data collected, the data is divided into two parts, in which 4000 data are randomly selected as the training sample set, and the remaining 1000 data are taken as the test sample set. Each data includes a total of 14 evaluation indicators, and the teaching quality assessment results are divided into five grades: excellent, good, middle, poor, worse, represented by 1,2,3,4 and 5 respectively. Part of the data is shown in Table 2.

\subsection{Model Implementation}

Firstly, the weight of teaching quality evaluation index is determine using AHP, and screening is conducted based on weights so as to obtain five indicators of teaching purposes [13], teaching tools and means; processing of difficult and focal points; completion of teaching plan; students' grasp of teaching content and so on. Then, normalization processing of these five indicators is carried out, and the normalized data and the actual results of the evaluation are formed into a new data set [14]. The training sample is input to BPNN for training, the training process shown in Fig. (3) $[15,16]$. 
Table 2. The teaching quality evaluation data of "data structure" in heilongjiang bayi agricultural university.

\begin{tabular}{|c|c|c|c|c|c|c|c|}
\hline No. & $\mathbf{x}_{1}$ & $\mathbf{x}_{2}$ & $\mathbf{x}_{3}$ & $\mathbf{x}_{4}$ & $\ldots$ & $\mathbf{x}_{14}$ & $\mathbf{y}$ \\
\hline 1 & 92 & 90 & 87 & 94 & $\ldots$ & 92 & 1 \\
\hline 2 & 74 & 67 & 60 & 64 & $\ldots$ & 69 & 4 \\
\hline 3 & 97 & 96 & 93 & 90 & $\ldots$ & 95 & 1 \\
\hline 4 & 88 & 90 & 90 & 82 & $\ldots$ & 98 & 1 \\
\hline 5 & 82 & 87 & 85 & 88 & $\ldots$ & 85 & 3 \\
\hline 6 & 93 & 95 & 97 & 90 & $\ldots$ & 95 & 1 \\
\hline 7 & 81 & 83 & 85 & 88 & $\ldots$ & 89 & 2 \\
\hline 8 & 83 & 82 & 84 & 88 & $\ldots$ & 85 & 2 \\
\hline 9 & 52 & 50 & 57 & 54 & $\ldots$ & 52 & 5 \\
\hline$\ldots$ & $\ldots$ & $\ldots$ & $\ldots$ & $\ldots$ & $\ldots$ & $\ldots$ & .. \\
\hline 500 & 89 & 80 & 85 & 84 & $\ldots$ & 87 & 2 \\
\hline
\end{tabular}

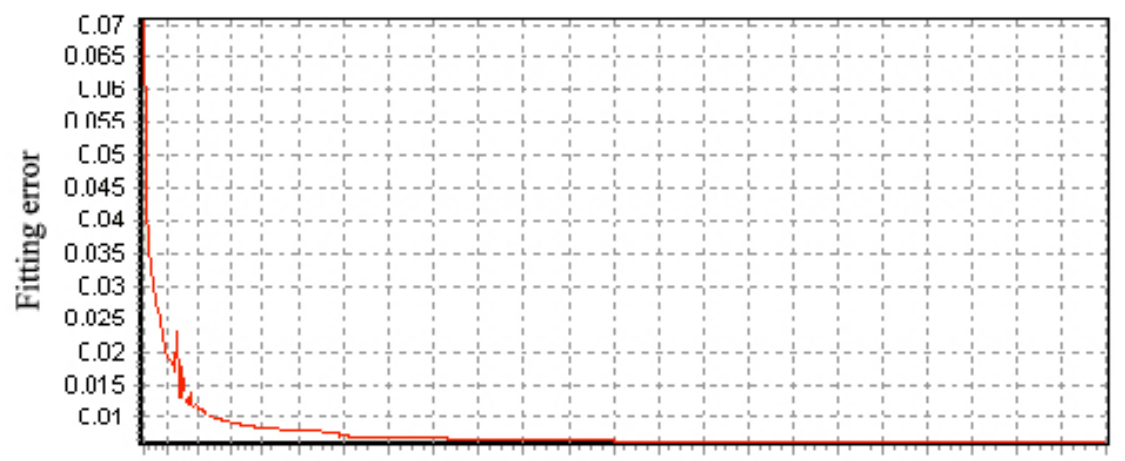

$119447095126^{\prime} 631992352^{7} 23083453814^{7} 45<49[527563600636672709745782$

Iterations

Fig. (3). Learning process of BPNN.

Test data of teaching quality in Anhui Vocational and Technical College are evaluated using the best established teaching quality evaluation model [17], and the obtained evaluation accuracy arrives at $98.5 \%$, with very high precision, and the results show that, teaching quality assessment method based on AHP-BPNN in this paper is effective and feasible.

\subsection{Comparison with Performance of Other Evaluation Models}

In order to detect the strengths and weaknesses of performance of evaluation models, comparative experiments are made in models of BPNN, AHP, MLR and AHP-MLR, and evaluation accuracy is taken as measure of the model, contrasting results shown in Table 3 .

From contrasting results in Table $\mathbf{3}$, the accuracy of evaluating teaching quality with combined model AHP-MLR and AHP-BPNN is higher than that of single model, which is mainly because the combined model uses the advantages of single model, realizing the complementary advantages, which has effectively improved the accuracy evaluation of teaching quality.
Table 3. Comparison of performance of each evaluation model.

\begin{tabular}{|c|c|}
\hline Evaluation Model & Precision \\
\hline \hline MLR & $85.6 \%$ \\
\hline AHP & $89.7 \%$ \\
\hline BPNN & $92.9 \%$ \\
\hline AHP-MLR & $91.3 \%$ \\
\hline AHP-BPNN & $98.5 \%$ \\
\hline
\end{tabular}

At the same time, the chart 3 shows that the evaluation results of BPNN are better than those of MLR and AHP, which is mainly because the modeling of neural network is conducted based on nonlinearity, with capabilities of intelligent learning and classification, while MLR and AHP is based on linear modeling, the non-linear relationship that fails to reflect the teaching quality evaluation index and evaluation rating very well. Therefore, BPNN is superior to other linear models. 
Furthermore, AHP-BPNN has the most accurate evaluation, indicating that the use of AHP to analyze evaluation index and to select the most important indicator of the evaluation results, and then the use of BPNN with strong non-linear predictive ability to implement teaching quality evaluation has made the best of strengths of both, and the both has sped up the efficiency and precision of teaching quality evaluation, able to conduct possible to effective classification and evaluation of teaching quality [17].

\section{CONCLUSION}

As for the issue of teaching quality evaluation, a method of teaching quality evaluation based on AHP-BPNN is proposed and performance testing is performed through simulation experiments, and the following conclusions are obtained. The use of NN to evaluate complex the teaching quality and use of AHP to screen the importance index not only simplifies the model structure of the neural network, while improving the evaluation accuracy of teaching quality, thereby dramatically reducing computation time of model and improving operational efficiency of teaching quality evaluation system. Simulation results show that for evaluation of teaching quality based on AHP-BPNN model, the evaluation results are more scientific and accurate, with good prospects in teaching management.

\section{CONFLICT OF INTEREST}

The authors confirm that this article content has no conflict of interest.

\section{ACKNOWLEDGEMENTS}

The research is supported by Cultivation Fund Project of Heilongjiang Bayi Agricultural University No. XRW2014-17.

\section{REFERENCES}

[1] A. Tek, B. Laurent, M. Piuzzi, L. Zhihan, M. Chavent, M. Baaden, and O. Delalande, "Advances in human-protein interactioninteractive and immersive molecular simulations," Biochemistry, Genetics and Molecular Biology"Protein-Protein InteractionsComputational and Experimental Tools', pp. 27-65, 2012.

D. Jiang, Z. Xu, P. Zhang, and T. Zhu, "A transform domain-based anomaly detection approach to network-wide traffic," Journal of Network and Computer Applications, vol. 40, no. 2, pp. 292-306, 2014.

[3] D. Jiang, Z. Xu, W. Li, and Z. Chen, "Network coding-based energy-efficient multicast routing algorithm for multi-hop wireless networks," Journal of Systems and Software, vol. 14, no. 2, pp. 101-112, 2015.

[4] Y. Geng, J. He, K. Pahlavan, "Modeling the effect of human body on TOA based indoor human tracking with Wrist Mounted Sensor" International Journal of Wireless Information Networks. vol. 20 no. 4, pp. 306-317, 2013.

[5] L. Zhang, B. He, J. Sun, M. Lai, L. Zhihan, "Double image multiencryption algorithm based on fractional chaotic time series," Journal of Computational and Theoretical Nanoscience, vol. 29, no. 2, pp. 248-256, 2015

[6] S. Li, Y. Geng, J. He, K. Pahlavan, "Analysis of three-dimensional maximum likelihood algorithm for capsule endoscopy localization," International Conference on Biomedical Engineering and Informatics (BMEI), pp. 721-725, 2012.

[7] S. Zhang, and H. Jing, "Fast log-Gabor-based nonlocal means image denoising methods," IEEE International Conference on Image Processing (ICIP), pp. 2724-2728, 2014.

[8] W. Luo, Z. Wang, L. Zhihan, "Method to acquire a complete road network in high-resolution remote sensing image based on tensor voting algorithm," Excli Journal, vol. 14, no. 2, pp. 101-112, 2015.

[9] L. Xiaoming, L. Zhihan, B. Zhang, L. Yin, W. Wang, S. Feng, and $\mathrm{J}$. Hu, "Traffic management and forecasting system based on $3 \mathrm{~d}$ gis cluster," IEEE/ACM International Symposium on Cloud and Grid Computing (CCGrid), 2015

[10] L. Xiaoming, L. Zhihan, B. Zhang, L. Yin, W. Wang, S. Feng, and J. Hu, "XEarth: A 3D GIS platform for managing massive city information," IEEE International Conference on Computational Intelligence and Virtual Environments for Measurement Systems and Applications(CIVEMSA), 2015.

[11] Y. Geng, J. Chen, K. Pahlavan, "Motion detection using RF signals for the first responder in emergency operations: A PHASER project," IEEE International Symposium on Personal Indoor and Mobile Radio Communications (PIMRC), 2013.

[12] J. Yang Z. Ding F. Guo and H. Wang, " Multiview image rectification algorithm for parallel camera arrays," Journal of Electronic Imaging, vol. 23, no. 3. pp. 1-33, 2013.

[13] J Yang, Y. Liu, Q. Meng, and R. Chu, "Objective evaluation criteria for stereo camera shooting quality under different shooting parameters and shooting distances, Sensors Journal, vol. 1, no. 99 pp. 1-15, 2013.

[14] L. Zhihan, A. Halawani, S. Feng, S. Rehman, and H. Li, "Multimodal hand and foot gesture interaction for handheld devices," ACM Transactions on Multimedia Computing, Communications, and Applications, vol. 11, no. 1, pp. 10-15, 2014.

[15] L. Zhihan, A. Halawani, S. Feng, S. Rehman, and H. Li, "Touchless interactive augmented reality game on vision based wearable device," IEEE International Symposium on Personal and Ubiquitous Computing, 2015.

[16] L. Zhihan, A. Tek, F. D. Silva, C. Empereur-Mot, M. Chavent, and M. Baaden, "Game on, science-how video game technology may help biologists tackle visualization challenges," PloS One, vol. 8, no. 3, pp. e57990- e57998, 2013.

[17] L. Zhihan, "Wearable smartphone: wearable hybrid framework for hand and foot gesture interaction on smartphone," IEEE International Workshop on Wearable Computer Vision Systems at International Conference on Computer Vision, 2013.

Received: June 10, 2015
(C) Yan and Bin; Licensee Bentham Open.

Revised: July 29, 2015

Accepted: August 15, 2015

This is an open access article licensed under the terms of the (https://creativecommons.org/licenses/by/4.0/legalcode), which permits unrestricted, noncommercial use, distribution and reproduction in any medium, provided the work is properly cited. 